\title{
Time and Intervention Effects of Daily Almond Intake on the Changes of Lipid Profile and Body Composition Among Free-Living Healthy Adults
}

\author{
Yanan Liu, ${ }^{1}$ Hyo-Jeong Hwang, ${ }^{2}$ Hyun-Sook Kim, and Hyunjin Park ${ }^{2}$ \\ ${ }^{I}$ Department of Food and Nutrition, College of Human Ecology, Sookmyung Women's University, Seoul, Korea. \\ ${ }^{2}$ ICAN Nutrition Education and Research, Seoul, Korea.
}

\begin{abstract}
Favorable health benefits of almond have been shown in several previous studies. However, repeated measures, randomized, controlled trials to investigate the changes due to almond intake based on the time effects have not yet been reported. The current study was conducted to evaluate the effects of daily almond intake on changes in body composition and lipid profiles for 20 weeks with four measurements among healthy adults. Participants in the almond group showed favorable changes on blood lipid profiles, including levels of triglycerides (TG), total cholesterol (TC), low-density lipoprotein cholesterol (LDL-C), and non-high-density lipoprotein (non-HDL-C) after consuming $56 \mathrm{~g}$ of almond per day for 20 weeks compared with those at baseline. At week 20, subjects in the almond group showed significantly decreased TC, LDL-C, nonHDL-C, TG, body fat mass, and waist-hip ratio compared with those of the control group who consumed isocaloric control food. The mixed model also confirmed that there were significant time effects in several bioimpedance indicators (i.e., total body protein, fat-free mass, etc.) and all of the lipid profile parameters in the almond group. These results confirm the effects of lipid-lowering and modifying body composition of almond consumption. In addition, our results suggest that the measuring time points would be critical to capture the effects of dietary intervention.
\end{abstract}

\section{KEYWORDS: • body composition $\bullet$ daily almond intake $\bullet$ lipid profile $\bullet$ mixed model $\bullet$ RCT $\bullet$ repeated measure}

\section{INTRODUCTION}

$\mathbf{E}$ PIDEMIOLOGICAL EVIDENCE suggests that the intake of tree nuts, including almond, may provide various health benefits especially for reducing the cardiovascular disease risk factors. ${ }^{1,2}$ According to a recent meta-analysis of 27 randomized, controlled trials using as an intervention by Musa-Veloso et al. ${ }^{3}$ almond consumption was associated with a significant reduction in total cholesterol (TC) $(P<.001)$, low-density lipoprotein cholesterol (LDL-C) $(P=.001)$, and triglycerides $(\mathrm{TG})(P=.042)$, and it had no effects on high-density lipoprotein (HDL-C) $(P=.207)$. More than half of the studies, including the meta-analysis, were randomized, parallel clinical trials mainly without a control group whereby the duration of the almond intervention ranged from 4 to 12 weeks. Longer term studies,

Trial Registration: This trial was registered at ClinicalTrials.gov as NCT03014531.

Manuscript received 7 April 2017. Revision accepted 19 November 2017.

Address correspondence to: Hyun-Sook Kim, PhD, Department of Food and Nutrition, College of Human Ecology, Sookmyung Women's University, Chungpa-Dong 2-Ka, Yongsan-Ku, Seoul 04310, Republic of Korea, E-mail: hskim@sookmyung.ac.kr or Hyunjin Park, PhD, ICAN Nutrition Education and Research, 20, Gukjegeumyung-ro, Yeongdeungpo-gu, Seoul 07327, Republic of Korea, E-mail: gracepark06@gmail.com

C Yanan Liu et al., 2018; Published by Mary Ann Liebert, Inc. This is an Open Access article distributed under the terms of the Creative Commons Attribution License, which permits unrestricted use, distribution, and reproduction in any medium, provided the original work is properly cited.
Wien et al. conducted a 24-week study to evaluate the effect of an almond-enriched or complex carbohydrate-enriched formula-based low-calorie diet on anthropometric data, body composition, and metabolic parameters in a weight reduction program in overweight and obese adults. The results showed significantly greater reductions in body weight, body mass index (BMI), waist circumference, and fat mass among overweight or obese subjects who consumed $84 \mathrm{~g}$ of almonds compared with those who did not consume almonds at all. ${ }^{4}$ Oleic acid, phenolic acids, phytosterols, and phytochemicals in almonds might collectively contribute to these cardioprotective qualities..$^{5,6}$

Even with the well-known favorable effects of consuming almond on a regular basis, there still has uncertainty whether two measuring points (i.e., pre- and postintervention) would explain the effects fully or might need additional measurements to examine the changes throughout a defined period. According to the repeated measures study with walnuts by Tapsell et al. ${ }^{7}$ metabolic and anthropometric parameters of participants seem to fluctuate throughout the trial period. With five measurements every 3 months during a year, the authors observed fluctuation of cholesterol levels during the trial. They also found that the weight loss and changes of body composition might be achieved at different time points.

Thus, we sought to investigate the effects of daily almond intake for 20 weeks on body composition and blood lipid profiles among healthy adults using a randomized controlled 
intervention trial. We particularly applied four times repeated measures to evaluate the time and interaction effects on changes of the parameters.

\section{MATERIALS AND METHODS}

\section{Subjects}

Participants in current analysis were part of the original study. ${ }^{8}$ In the original study, a total of 169 subjects completed 16 weeks trials and 85 out of 169 subjects continued participating in 4 additional weeks. They were nonsmokers, 20 39 years of age, and had no clinical symptoms and significant changes of body weight during the last 6 months. Their BMIs were $17-30 \mathrm{~kg} / \mathrm{m}^{2}$. For more details about recruiting subjects, see the original study. This study was conducted according to the guidelines laid down in the Declaration of Helsinki, and all procedures involving human subjects were approved by the Ethics Committee of Sookmyung Women's University, Seoul, Korea (SMWU-1407-BR-006). Written informed consent was obtained from all study subjects.

\section{Study design}

This study was a 20-week, randomized, controlled trial with four serial measurements $(0,8,16$, and 20 weeks of intervention). Current study is an extension of the original one by adding one additional measurement at week 20. Participants in the almond group $(n=57)$ consumed $56 \mathrm{~g}$ of almonds per day and participants in the control group $(n=28)$ were provided with high-carbohydrate control food items that had a similar number of calories as $56 \mathrm{~g}$ of almonds. Subjects were instructed to maintain their habitual dietary intake and usual level of physical activity and avoid consuming any additional nuts or nut products throughout the study.

\section{Compliance and dietary assessment}

Good compliance was defined as $>80 \%$ adherence to the treatment. The subjects were excluded if they had a compliance $<80 \%$. Details for measuring compliance were written in the original study. The 3-day diet records, including two consecutive weekdays and one weekend day, were assessed before the trial and at the last week of the trial. A registered dietitian provided detailed instructions on how to fill out a diet record and report their diet by taking pictures and sending the information through text messages. All dietary records were analyzed to provide an estimation of the daily average energy and nutrient intake using CAN-Pro 4.0 software (Korean Nutrition Society).

\section{Measurements}

All measurements were conducted with participants going barefoot and wearing light clothing for the baseline (week 0) and weeks 8, 16, and 20. Waist and hip circumference measurements were measured by trained technical staff using a measuring tape (SECA-201; SECA, Ltd., Hamburg, Germany). Waist circumferences were obtained at the mid-point between the lowest rib and the iliac crest to the nearest $0.1 \mathrm{~cm}$ after inhalation and exhalation. Hip cir- cumferences were rounded to the nearest $0.1 \mathrm{~cm}$ at the widest part between the waist and knees. Weight, while fasting, was measured and rounded to the nearest $0.1 \mathrm{~kg}$, and height was rounded to the nearest $0.1 \mathrm{~cm}$ using a stadiometer (BSM330; Biospace, Co., Ltd., Seoul, Korea). Blood pressure was measured on the right arm using an upload blood pressure monitor (BPBIO320S; Biospace, Co., Ltd.) with participants in a comfortably seated position after at least a 5-min rest. Body composition was assessed through multifrequency whole-body bioimpedance measurement using InBody 620 (Biospace, Co., Ltd.). The results included body composition analysis (total body water, protein, and body fat mass), muscle-fat analysis (body weight, skeletal muscle mass, and body fat mass), and obesity analysis [body fat percentage and body fat mass index (BMI) (calculated as weight $(\mathrm{kg})$ divided by height in meters $(\mathrm{m})$ squared)].

\section{Blood analyses}

After a 12-h fast, blood samples were taken at the baseline time point (week 0 ) and at weeks 8,16 , and 20 by standard venipuncture. Serum TC and TG levels were measured by the enzymatic colorimetric method using a Cobas 8000 c702 chemistry analyzer (Roche Diagnostics, Mannheim, Germany). HDL-C and LDL-C levels were determined through homogeneous enzymatic colorimetry. Non-HDL-C levels were calculated from the TC and HDL-C using the following formula: non-HDL-C $(\mathrm{mg} / \mathrm{dL})=$ TC-HDL-C. ${ }^{9}$

\section{Statistical analyses}

In the current analyses, data obtained from two different almond groups were aggregated together and set as almond group. Paired $t$-test was used for comparison of the measurements before and after the study in almond and control groups. Comparison between almond group and control group was performed using independent sample $t$-test at both baseline and week 20. Comparison between almond group and control group for changes in anthropometric measurements, bioimpedance parameters, and blood lipid profiles from baseline to week 20 were performed using independent sample $t$-test. MIXED procedure was performed to test the effect of almond consumption on bioimpedance analysis measurements and serum lipid profiles. These measures and models accounted for the within-subject variation in parameters, including gender and the intervention groups as fixed effect terms. All data were expressed as mean with standard deviations, unless otherwise specified. All statistical analyses were performed using SAS (version 9.3; SAS Institute, Cary, NC).

\section{RESULTS}

\section{Basic characteristics and dietary analyses}

Baseline characteristics of the participants are presented in Table 1. Between-group differences for anthropometric and body composition measurements were not observed, but the levels of TG $(P=.001)$, TC $(P=.041)$, LDL-C $(P=.024)$, nonHDL-C $(P=.020)$, and very LDL-C (VLDL-C) $(P=.001)$ 
Table 1. Baseline Characteristics of the Participants

\begin{tabular}{|c|c|c|c|}
\hline & Almond $(\mathrm{n}=57)(M 31 / F 26)$ & Control $(\mathrm{n}=28)(M 14 / F$ 14 $)$ & $\mathrm{P}$ \\
\hline Age (years) & $26.96 \pm 5.22^{\mathrm{a}}$ & $26.14 \pm 5.40$ & .502 \\
\hline Systolic blood pressure (mmHg) & $124.58 \pm 13.79$ & $126.29 \pm 16.76$ & .619 \\
\hline Diastolic blood pressure (mmHg) & $74.65 \pm 8.64$ & $74.61 \pm 14.35$ & .987 \\
\hline Waist $(\mathrm{cm})$ & $76.81 \pm 9.21$ & $74.61 \pm 8.34$ & .289 \\
\hline Hip $(\mathrm{cm})$ & $97.68 \pm 6.3$ & $95.68 \pm 5.95$ & .164 \\
\hline Height $(\mathrm{cm})$ & $166.86 \pm 9.41$ & $169.16 \pm 8.88$ & .284 \\
\hline Weight (kg) & $64.48 \pm 13.21$ & $62.27 \pm 11.75$ & .455 \\
\hline BMI $\left(\mathrm{kg} / \mathrm{m}^{2}\right)$ & $23 \pm 3.17$ & $21.66 \pm 3.08$ & .068 \\
\hline $\mathrm{TC}(\mathrm{mg} / \mathrm{dL})$ & $177.11 \pm 29.58$ & $162.64 \pm 31.54$ & .041 \\
\hline HDL-C (mg/dL) & $58.68 \pm 13.45$ & $61.54 \pm 16.53$ & .397 \\
\hline TC:HDL-C & $3.2 \pm 1.09$ & $2.79 \pm 0.81$ & .080 \\
\hline LDL-C (mg/dL) & $111.12 \pm 27.44$ & $96.29 \pm 29.23$ & .024 \\
\hline HDL:LDL-C & $0.57 \pm 0.23$ & $0.71 \pm 0.31$ & .041 \\
\hline non-HDL-C (mg/dL) & $118.42 \pm 31.54$ & $101.11 \pm 31.5$ & .020 \\
\hline TG $(\mathrm{mg} / \mathrm{dL})$ & $91.58 \pm 51.33$ & $64.82 \pm 21.76$ & .001 \\
\hline VLDL-C (mg/dL) & $18.32 \pm 10.27$ & $12.96 \pm 4.35$ & .001 \\
\hline Total body protein $(\mathrm{kg})$ & $9.31 \pm 2.22$ & $9.37 \pm 2.07$ & .901 \\
\hline Body fat mass $(\mathrm{kg})$ & $17.23 \pm 6.42$ & $14.63 \pm 6.86$ & .089 \\
\hline Body fat percentage $(\%)$ & $26.79 \pm 8.22$ & $23.4 \pm 9.12$ & .089 \\
\hline Fat free mass $(\mathrm{kg})$ & $47.25 \pm 10.99$ & $47.64 \pm 10.36$ & .874 \\
\hline Soft lean mass $(\mathrm{kg})$ & $44.43 \pm 10.41$ & $44.88 \pm 9.85$ & .850 \\
\hline Skeletal muscle mass $(\mathrm{kg})$ & $26.11 \pm 6.68$ & $26.32 \pm 6.19$ & .890 \\
\hline Visceral fat level ${ }^{\mathrm{b}}$ & $6.86 \pm 3.27$ & $5.68 \pm 3.48$ & .129 \\
\hline Waist-Hip ratio & $0.84 \pm 0.04$ & $0.83 \pm 0.06$ & .247 \\
\hline Basal metabolic rate (kcal) & $1390.56 \pm 237.49$ & $1398.93 \pm 223.97$ & .877 \\
\hline
\end{tabular}

${ }^{a}$ Values are mean \pm SD.

'bIndicator generated from bioimpedance analysis based on the estimated amount of fat surrounding internal organs in the abdomen (normal range: 1-9).

BMI, body mass index; F, female; HDL-C, high-density lipoprotein; LDL-C, low-density lipoprotein cholesterol; M, male; SD, standard deviation; TC, total cholesterol; TG, triglycerides; VLDL-C, very LDL-C.

Table 2. Dietary Analyses Results for Baseline and Week 20 of Almond and Control Groups

\begin{tabular}{|c|c|c|c|c|c|c|c|c|}
\hline \multirow[b]{2}{*}{ Variable } & \multicolumn{2}{|c|}{ Baseline } & \multirow[b]{2}{*}{$\mathrm{P}_{l}^{\mathrm{b}}$} & \multicolumn{2}{|c|}{ Week 20} & \multirow[b]{2}{*}{$\mathrm{P}_{2}$} & \multirow{2}{*}{$\begin{array}{c}\text { Almond } \\
\mathrm{P}_{3}\end{array}$} & \multirow{2}{*}{$\begin{array}{c}\text { Control } \\
\mathrm{P}_{4}\end{array}$} \\
\hline & Almond $(\mathrm{n}=56)$ & Control $(\mathrm{n}=28)$ & & Almond $(\mathrm{n}=56)$ & Control $(\mathrm{n}=28)$ & & & \\
\hline Energy (kcal) & $1632.25 \pm 392.14^{\mathrm{a}}$ & $1613.85 \pm 360.85$ & .836 & $1730.41 \pm 342.48$ & $1883.72 \pm 510.99$ & .159 & .136 & .015 \\
\hline Carbohydrate (g) & $220.93 \pm 58.01$ & $221.35 \pm 49.94$ & .974 & $212.55 \pm 48.43$ & $255.5 \pm 70.69$ & .006 & .357 & .021 \\
\hline Total fat $(\mathrm{g})$ & $54.37 \pm 17.88$ & $49.89 \pm 12.78$ & .192 & $70.79 \pm 16.31$ & $64.26 \pm 20.47$ & .116 & .000 & .000 \\
\hline Vegetable fat $(\mathrm{g})$ & $28.63 \pm 11.31$ & $25.63 \pm 8.96$ & .225 & $49.53 \pm 12.19$ & $38.5 \pm 12.1$ & .000 & .000 & .000 \\
\hline Animal fat $(\mathrm{g})$ & $25.74 \pm 11.17$ & $24.26 \pm 9.02$ & .544 & $21.26 \pm 11.3$ & $25.75 \pm 12.99$ & .106 & .018 & .767 \\
\hline Total protein $(\mathrm{g})$ & $63.11 \pm 17.2$ & $62.35 \pm 14.92$ & .842 & $65.49 \pm 15.07$ & $67.35 \pm 21.28$ & .682 & .382 & .211 \\
\hline Vegetable protein $(\mathrm{g})$ & $28.08 \pm 8.02$ & $25.97 \pm 6.06$ & .224 & $34.75 \pm 7.44$ & $29.38 \pm 8.93$ & .005 & .000 & .085 \\
\hline Animal protein $(\mathrm{g})$ & $35.03 \pm 13.8$ & $36.37 \pm 13.06$ & .670 & $30.74 \pm 12.15$ & $37.97 \pm 17.15$ & .028 & .033 & .593 \\
\hline Fiber $(\mathrm{g})$ & $14.55 \pm 4.49$ & $15.42 \pm 3.62$ & .378 & $18.83 \pm 4.73$ & $14.19 \pm 4.8$ & .000 & .000 & .220 \\
\hline Vitamin E (mg) & $15.22 \pm 5.86$ & $16.22 \pm 6.24$ & .474 & $27.92 \pm 7.23$ & $17.73 \pm 6.48$ & .000 & .000 & .359 \\
\hline Riboflavin (mg) & $1.1 \pm 0.37$ & $1.01 \pm 0.27$ & .226 & $1.35 \pm 0.28$ & $1.05 \pm 0.33$ & .000 & .000 & .596 \\
\hline Calcium (mg) & $429.25 \pm 187.34$ & $417.64 \pm 155.44$ & .778 & $482.14 \pm 147.28$ & $413.22 \pm 186.12$ & .068 & .093 & .923 \\
\hline Phosphorus (mg) & $916.17 \pm 249.6$ & $890.02 \pm 199.78$ & .631 & $1040.8 \pm 211.3$ & $913.3 \pm 250.09$ & .016 & .004 & .652 \\
\hline Magnesium (mg) & $62.66 \pm 27.4$ & $68.98 \pm 29.46$ & .334 & $193.05 \pm 49.78$ & $64.2 \pm 28.86$ & .000 & .000 & .538 \\
\hline Cholesterol (mg) & $348.76 \pm 158.28$ & $366.94 \pm 170.12$ & .630 & $307.95 \pm 137.73$ & $385.55 \pm 154.11$ & .022 & .084 & .531 \\
\hline Saturated fatty acid (g) & $10.06 \pm 6.51$ & $8.94 \pm 4.82$ & .421 & $10.04 \pm 5.87$ & $9.65 \pm 5.68$ & .777 & .980 & .608 \\
\hline MUFAs (g) & $11.91 \pm 7.36$ & $11.11 \pm 5.74$ & .613 & $26.32 \pm 8.51$ & $11.55 \pm 6.09$ & .000 & .000 & .782 \\
\hline PUFAs (g) & $8.89 \pm 4.55$ & $9.37 \pm 3.62$ & .630 & $13.19 \pm 4.02$ & $9.46 \pm 4.14$ & .000 & .000 & .923 \\
\hline
\end{tabular}

${ }^{a}$ Values are mean \pm SD.

${ }^{\mathrm{b}} P_{1}, P_{2}$ values resulted from independent sample $t$-test, and $P_{3}, P_{4}$ values resulted from paired $t$-test.

MUFAs, monounsaturated fatty acids; PUFAs, polyunsaturated fatty acids. 


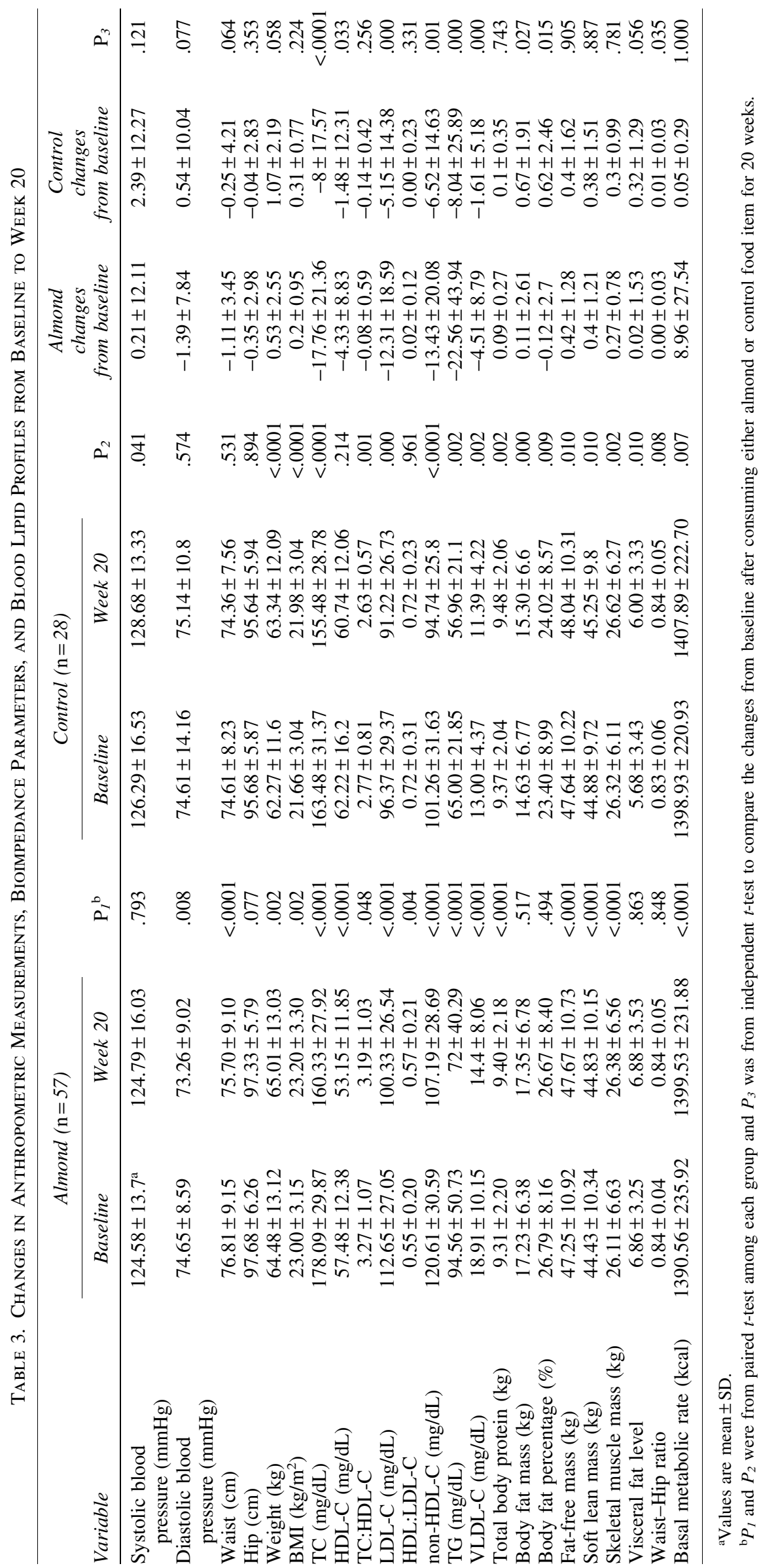


were significantly lower in the control group compared with those of the almond group.

According to the results of dietary analyses in Table 2, participants in almond group consumed significantly greater amount of vegetable protein $(P=.000)$, fiber $(P=.000)$, vitamin E $(P=.000)$, riboflavin $(P=.000)$, phosphorus $(P=.004)$, magnesium $(P=.000)$, monounsaturated fatty acids (MUFAs) $(P=.000)$, and polyunsaturated fatty acids (PUFAs) $(P=.000)$ compared with baseline after 20 weeks of consuming almonds. No significant changes in intake of energy between baseline and week 20 were observed $(P=.136)$. Participants in the control group consumed significantly higher energy $(P=.015)$ and greater amounts of carbohydrate $(P=.021)$ and total fat $(P=.000)$ during the trial compared with those at baseline and total protein intake were not significantly different between almond and control groups at baseline. However, at week 20, carbohydrate $(P=.006)$, animal protein $(P=.028)$, and cholesterol $(P=.022)$ intake of the almond group were lower than those of the control group, whereas intake of vegetable fat $(P=.000)$, vegetable protein $(P=.005)$, fiber $(P=.000)$, vitamin E $(P=.000)$, riboflavin $(P=.000)$, phosphorus $(P=.016)$, magnesium $(P=.000)$, MUFAs $(P=.000)$, and PUFAs $(P=.000)$ of the almond group were significantly higher. There were no significant differences between groups in energy intake $(P=.159)$.

\section{Comparisons of the changes from the baseline for bioimpedance parameters and blood lipid profiles}

Changes in anthropometric measurements, bioimpedance parameters, and blood lipid profiles from baseline to week 20 are shown in Table 3.

1. Within-group effects: Significantly decreased waist circumference $(P<.0001)$ was observed in the almond group even though their body weights were significantly increased $(P=.002)$. Subjects consuming almonds for 20 weeks showed significantly reduced levels of TC $(P<.0001)$, HDL-C $(P<.0001)$, TC: HDL-C $(P=.048)$, LDL-C $(P<.0001)$, non-HDL-C $(P<.0001)$, TG $(P<.0001)$, and VLDL-C $(P<.0001)$ compared with the values at baseline, whereas HDL:LDL-C $(P=.004)$ was significantly increased after consuming almond for 20 weeks. Total body protein, fat free mass, soft lean mass, skeletal muscle mass, and basal metabolic rate were significantly increased after 20 weeks of almond consumption $(P<.0001$ for all $)$. The control group also showed several significances. Body weight $(P<.0001)$ and BMI $(P<.0001)$ were significantly increased. Significant reduction on the levels of TC $(P<.0001)$, TC:HDL-C $(P=.001)$, LDL-C $(P=.000)$, non-HDL-C $(P<.0001)$, TG $(P=.002)$, and VLDL-C $(P=.002)$ were observed in control group as well.

2. Between-group effects: No significant differences in weight changes were found between control and almond groups at week 20. Compared with control group, participants in the almond group showed significantly greater reduction in serum TC $(-17.76 \pm 21.36, P<$
$.0001), \quad$ HDL-C $\quad(-4.33 \pm 8.83, \quad P=.033), \quad$ LDL-C $(-12.31 \pm 18.59, P=.000)$, non-HDL-C $(-13.43 \pm 20.08$, $P=.001)$, TG $(-22.56 \pm 43.94, P=.000)$, VLDL-C $(-4.51 \pm 8.79, P=.000)$, and body fat percentages $(-0.12 \pm 2.7, P=.015)$ after 20 weeks of almond intervention. Subjects in the control group showed significantly increased body fat mass $(0.67 \pm 1.91$, $P=.027)$ and waist-hip ratio $(0.01 \pm 0.03, P=.035)$ compared with those of the almond group.

\section{Results from mixed procedure}

Time, intervention, and interaction effects throughout the trial were analyzed using the mixed model (Table 4). The almond group showed a significant time effects for changes in diastolic blood pressure and waist circumference, several bioimpedance measurements, including total body protein, fat-free mass, soft lean mass, skeletal muscle mass, and basal metabolic rate, and all of lipid profile parameters. Significant interaction effects were observed for the changes in TC $(P=.009)$ and non-HDL-C $(P=.030)$. There were significant group effects for changes in HDL-C $(P=.008)$, TC:HDL-C $(P=.016)$, HDL:LDL-C $(P=.006)$, TG $(P=.036)$, and VLDL-C $(P=.036)$. There were no significant interactions and group effects for changes in anthropometric and bioimpedance measurements adjusted for gender.

\section{DISCUSSION}

As far as we know, there has been no repeated measures, randomized, controlled trial administering almonds for longer than 12 weeks. The original 16-week study was the first randomized controlled trial with repeated measurements and the current study, which is the extension with the fourth measurement at week 20 , provided additional information about the health benefits of almond consumption. This study revealed that the effect of dietary intervention appeared differently by times repeated measures and the changes of lipid profiles achieved at different time points. For example, TG levels of the almond group were gradually decreased throughout the trial period, whereas HDL-C levels were slightly increased at week 8 , and then decreased during rest of the trial. Subjects in the control group also showed fluctuation in lipid profile parameters during the trial, which may indicate the duration of the intervention and measuring time point would be crucial to capture the effects of certain intervention.

Interestingly, both almond and control groups showed significantly favorable results especially on their lipid profiles, which means intervention study effects might produce the positive effects even among healthy adults. These study effects were observed in several previous studies conducted by Wien et al. ${ }^{4}$ and Abazarfard et al. ${ }^{10}$ It might be the selfwillingness of the participants who seemed to be concerned about their health and healthy eating and/or healthy food. In the current study, the almond group showed significantly greater reductions in serum TC, LDL-C, non-HDL-C, TG, and VLDL-C compared with those of control group, even though participants in the control group also showed 


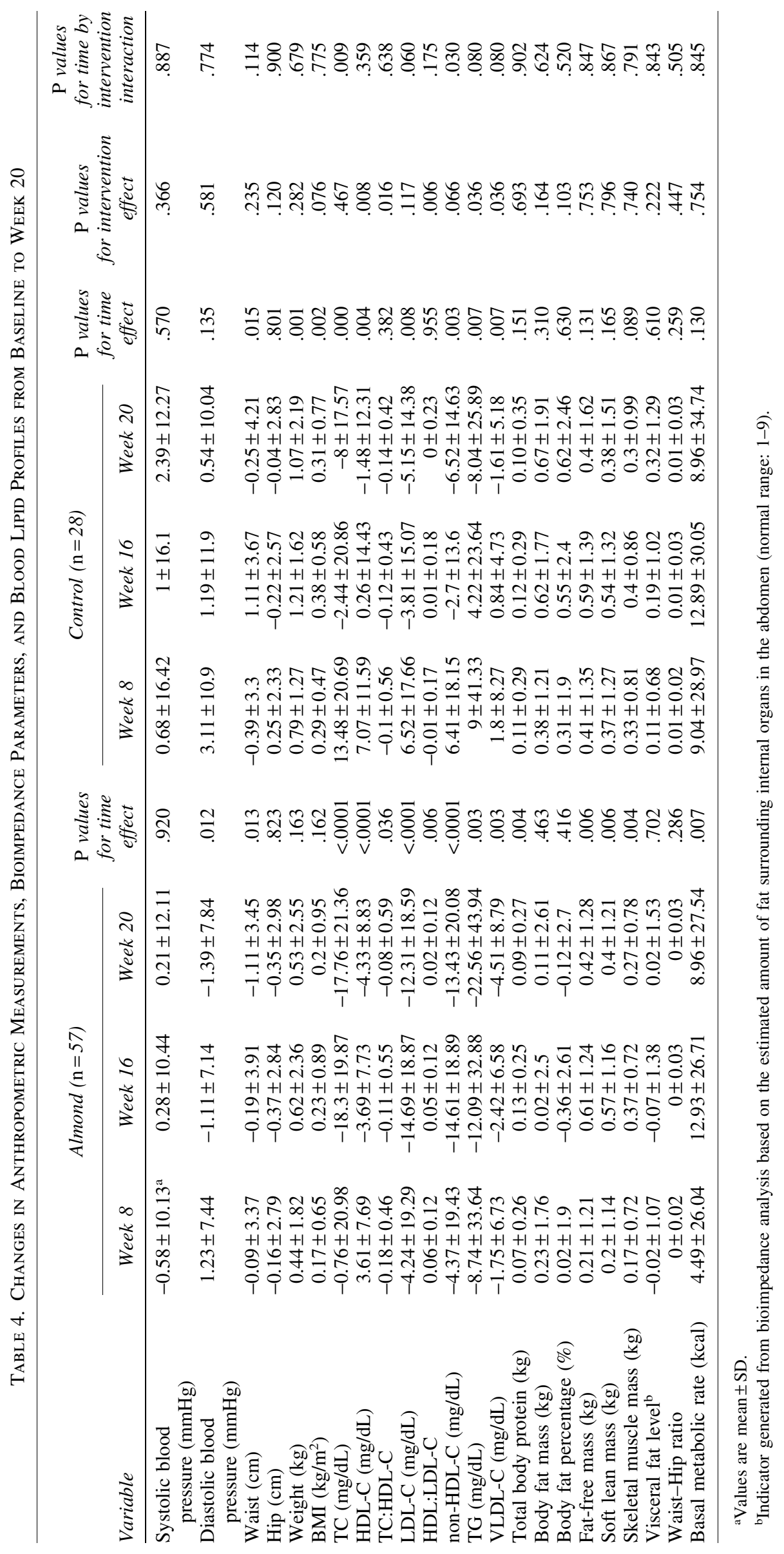


favorable changes for lipid profiles. These results suggest that protein, dietary fiber, vitamin E, MUFAs, and PUFAs in almond may collectively contribute to their cardioprotective qualities. ${ }^{6}$ These are similar to the recent meta-analysis report, which showed that consumption of almond decreases TC, LDL-C, and TG levels. Nutrient balance of almonds may affect the whole-day diet, which was reflected in the second dietary assessment. Carbohydrate intake of the almond group were lower than those of the control group, whereas intake of vegetable fat, vegetable protein, fiber, vitamin E, phosphorus, magnesium, MUFAs, and PUFAs of almond group were significantly higher. It should also be pointed out that the intake of vitamin $\mathrm{E}$ was increased 1.5 times, and magnesium and MUFAs were increased more than two times in almond group than control group. The stronger favorable changes in blood lipid levels were due to changes from baseline in nutrient intake that are known to influence blood cholesterol, such as protein, ${ }^{11}$ fiber, ${ }^{12}$ vitamin $\mathrm{E},{ }^{13}$ MUFAs, and PUFAs ${ }^{14}$ after consumption of almonds.

A significant decrease in HDL-C levels in both almond and control groups at week 20 was already reported in previous studies. In a meta-analysis of five randomized, 4-week intervention trials, investigators reported a trend toward a $0.05 \mathrm{mmol} / \mathrm{L}$ reduction in HDL-C associated with almond consumption. ${ }^{15}$ Based on Lovejoy et al. ${ }^{16}$ high-fat diets containing almonds result in the lowest concentrations of TC, but significant decreases in HDL-C in the subjects with type 2 diabetes and a significant decrease in HDL-C was also found in the healthy subjects. The mechanism by which almonds reduce HDL-C requires further research. Perhaps because most of the previous trials included healthy, ${ }^{17}$ hyperlipidemic, ${ }^{18}$ obese,${ }^{19}$ or diabetic ${ }^{16}$ individuals whose baseline HDL-C were not particularly low. However, a study conducted by Jamshed et al. ${ }^{20}$ to evaluate the effect of almond supplementation on low HDL-C in coronary artery disease patients indicated that almond supplementation can markedly improve low HDL-C, in addition to reducing other serum lipids. The lack of effects on HDL-C by almonds in the study may be explained by the characteristics of subjects. The participants included in this study were relatively young ( $26.69 \pm 5.26$ years of age) and healthy. Their serum lipid profiles especially HDL-C levels were within the healthy range at baseline and end of the trial. Although the serum cholesterol and LDL-C of the participants were within the normal ranges, the almond interventions further reduced lipidemia, which provides evidence of the beneficial effects of almond consumption in healthy adults.

Unlike the lipid profiles, results from bioimpedance measurement did not show the significance between groups. Almond consumption for 20 weeks influenced total body protein, fat-free mass, and skeletal muscle mass; however, these are shown only within the almond group. Even though there were no differences between almond and control groups for bioimpedance parameters, the current study still confirmed that the almond intake may make an impact on the diet and nutrient composition and present various health benefits.

The main strengths of the current study include sufficient study duration and time-series measurement. Major out- comes of the study may be provided based on the study design. In addition, our subjects were under free-living condition and had no restrictions about their everyday lives. This means that the results may be applicable to actual life situations. We also had participants for control who consumed isocaloric high-carbohydrate food. Since several previous studies have been conducted without control food, ${ }^{21-23}$ it was questionable whether the effects of almond intake were the result of consuming almonds or reducing any food from subjects' habitual diet. Randomized controlled trials like the current study may give in-depth information by having a control group. Even with these strengths, there are still limitations. The isocaloric control food contained higher percentages of carbohydrates compared with almonds, which means it does not match the macronutrient composition of almonds. Further studies providing a control food item with matched macronutrient and fatty acid composition are needed. Almond and control food items we provided were not able to be blinded, since our participants consumed whole almonds or cookies. In addition, since the subjects were dropped out at the point of their compliances being under $80 \%$, it was not possible to conduct intent-to-treat analysis. The current study has a sufficient duration to discern the effects of almonds on blood profiles but a longer-term study, over a year, with a parallel, randomized, controlled trial applying time-series measurements would provide additional valuable information about daily almond consumption.

In conclusion, the effects of almond appeared differently when using times repeated measures and the changes of lipid profiles achieved at different time points. Continuous consumption of almond for 20 weeks may improve serum lipid profiles and body composition in healthy young adults.

\section{ACKNOWLEDGMENTS}

The authors thank W. Jeon, E. Song, H. Jung, J. Jang, and E. Jung for their technical support. Funding was provided by the Almond Board of California.

\section{AUTHORS' CONTRIBUTIONS}

Y.L., H.P., and H.S.K. designed research; Y.L., H.P., and H.S.K. conducted research; Y.L., H.J.H., and H.P. analyzed data; Y.L. and H.P. wrote the article; and H.P. and H.S.K. had primary responsibility for final content.

\section{AUTHOR DISCLOSURE STATEMENT}

No conflicts of interest, financial or otherwise, are declared by the author(s).

\section{REFERENCES}

1. Ros E: Nuts and novel biomarkers of cardiovascular disease. Am J Clin Nutr 2009;89:1649S-1656S.

2. Ros E: Nuts and CVD. Br J Nutr 2015;113(Suppl 2):S111-S120.

3. Musa-Veloso K, Paulionis L, Poon T, Lee HY: The effects of almond consumption on fasting blood lipid levels: A systematic review and meta-analysis of randomised controlled trials. $J$ Nutr Sci 2016;5:e34. 
4. Wien MA, Sabate JM, Ikle DN, Cole SE, Kandeel FR: Almonds vs complex carbohydrates in a weight reduction program. Int $J$ Obes (Lond) 2003;27:1365-1372.

5. Bolling BW, Chen CY, McKay DL, Blumberg JB: Tree nut phytochemicals: Composition, antioxidant capacity, bioactivity, impact factors. A systematic review of almonds, Brazils, cashews, hazelnuts, macadamias, pecans, pine nuts, pistachios and walnuts. Nutr Res Rev 2011;24:244-275.

6. Berryman CE, Preston AG, Karmally W, Deckelbaum RJ, KrisEtherton PM: Effects of almond consumption on the reduction of LDL-cholesterol: A discussion of potential mechanisms and future research directions. Nutr Rev 2011;69:171-185.

7. Tapsell LC, Batterham MJ, Teuss G, et al.: Long-term effects of increased dietary polyunsaturated fat from walnuts on metabolic parameters in type II diabetes. Eur J Clin Nutr 2009;63:10081015.

8. Liu Y, Hwang HJ, Ryu H, Lee YS, Kim HS, Park H: The effects of daily intake timing of almond on the body composition and blood lipid profile of healthy adults. Nutr Res Pract 2017;11:479-486.

9. Wu L, Piotrowski K, Rau T, et al.: Walnut-enriched diet reduces fasting non-HDL-cholesterol and apolipoprotein B in healthy Caucasian subjects: A randomized controlled cross-over clinical trial. Metabolism 2014;63:382-391.

10. Abazarfard Z, Salehi M, Keshavarzi S: The effect of almonds on anthropometric measurements and lipid profile in overweight and obese females in a weight reduction program: A randomized controlled clinical trial. J Res Med Sci 2014;19:457-464.

11. Ruiz Ruiz JC, Betancur Ancona DA, Segura Campos MR: Bioactive vegetable proteins and peptides in lipid-lowering; nutraceutical potential. Nutr Hosp 2014;29:776-784.

12. Satija A, Hu FB: Cardiovascular benefits of dietary fiber. Curr Atheroscler Rep 2012;14:505-514.

13. Saggini A, Anogeianaki A, Angelucci D, et al.: Cholesterol and vitamins: Revisited study. J Biol Regul Homeost Agents 2011;25: 505-515.
14. McNamara DJ: Dietary fatty acids, lipoproteins, and cardiovascular disease. Adv Food Nutr Res 1992;36:253-351.

15. Phung OJ, Makanji SS, White CM, Coleman CI: Almonds have a neutral effect on serum lipid profiles: A meta-analysis of randomized trials. J Am Diet Assoc 2009;109:865-873.

16. Lovejoy JC, Most MM, Lefevre M, Greenway FL, Rood JC: Effect of diets enriched in almonds on insulin action and serum lipids in adults with normal glucose tolerance or type 2 diabetes. Am J Clin Nutr 2002;76:1000-1006.

17. Sabate J, Haddad E, Tanzman JS, Jambazian P, Rajaram S: Serum lipid response to the graduated enrichment of a step I diet with almonds: A randomized feeding trial. Am J Clin Nutr 2003; 77:1379-1384.

18. Jenkins DJ, Kendall CW, Marchie A, et al.: Dose response of almonds on coronary heart disease risk factors: Blood lipids, oxidized low-density lipoproteins, lipoprotein(a), homocysteine, and pulmonary nitric oxide: A randomized, controlled, crossover trial. Circulation 2002;106:1327-1332.

19. Foster GD, Shantz KL, Vander Veur SS, et al.: A randomized trial of the effects of an almond-enriched, hypocaloric diet in the treatment of obesity. Am J Clin Nutr 2012;96:249-254.

20. Jamshed H, Sultan FA, Iqbal R, Gilani AH: Dietary almonds increase serum HDL cholesterol in coronary artery disease patients in a randomized controlled trial. J Nutr 2015;145:2287-2292.

21. Tan SY, Mattes RD: Appetitive, dietary and health effects of almonds consumed with meals or as snacks: A randomized, controlled trial. Eur J Clin Nutr 2013;67:1205-1214.

22. Sweazea KL, Johnston CS, Ricklefs KD, Petersen KN: Almond supplementation in the absence of dietary advice significantly reduces C-reactive protein in subjects with type 2 diabetes. $J$ Funct Foods 2014;10:252-259.

23. Hull S, Re R, Chambers L, Echaniz A, Wickham MS: A midmorning snack of almonds generates satiety and appropriate adjustment of subsequent food intake in healthy women. Eur $J$ Nutr 2015;54:803-810. 